\title{
The Basic Physics of Waves, Soundwaves, and Shockwaves for Erectile Dysfunction
}

\author{
Jonathan Elliott Katz, MD1, Raul Ivan Clavijo, MD², Paul Rizk, MD', Ranjith Ramasamy, MD1 \\ 1. University of Miami Department of Urology, Miami, USA \\ 2.University of California at Davis Department of Urology, Davis, USA
}

\author{
Keywords \\ Extracorporeal Shockwave Therapy; Erectile Dysfunction
}

\section{Introduction:}

Over the past decade, Li-ESWT (Low-intensity Extracorporeal Shockwave Therapy) has emerged as a controversial treatment modality for erectile dysfunction (ED). In 2012 Vardi et. al. demonstrated significant improvement in erectile function in men treated with $\mathrm{Li}$ ESWT versus placebo. [1] In the ensuing years, many international trials have investigated Li-ESWT for ED, but the results have been equivocal and mired in critiques regarding heterogeneity of these studies. Nonetheless, there are several high quality randomized clinical trials [1-4] and multiple meta-analyses that demonstrate a clinically significant benefit to Li-ESWT in the treatment of ED. [5-7]

Despite this evidence, Li-ESWT is not Food and Drug Administration (FDA) approved and is considered investigational in the United States (US), and available to patients under clinical trial protocols. [8] Unfortunately, different clinical trials have employed different treatment protocols with different devices, and it remains unclear if these devices have equivalent treatment efficacy. Compounding this is skepticism regarding the mechanism of action of Li-ESWT. For several years, urologists have harnessed the biologic effects of shockwaves with ESWL (extracorporeal shock wave lithotripsy). Although most urologists have a general framework of how a shock can lead to break-up of stones, it is difficult to conceptualize how low energy shockwaves can lead to enhancement of erectile function.

To appreciate the science as well as subtleties in the evidence for and against the variety of different Li-ESWT devices available for the treatment of ED, it is imperative to understand

Corresponding Author Name: Ranjith Ramasamy, ramasamy@miami.edu.

Publisher's Disclaimer: This is a PDF file of an unedited manuscript that has been accepted for publication. As a service to our customers we are providing this early version of the manuscript. The manuscript will undergo copyediting, typesetting, and review of the resulting proof before it is published in its final form. Please note that during the production process errors may be discovered which could affect the content, and all legal disclaimers that apply to the journal pertain.

Conflict of Interest: Ranjith Ramasamy was provided with a MoreNova shockwave device by DirexGroup (the product manufacturer) and received financial support from the Barton Weiss Men's Health Initiative. The remaining authors have nothing to disclose 
the underlying physics and biological effects behind low intensity shockwaves. In this article we explain the physics of shockwaves by establishing a foundation regarding the basics of waves, specifically soundwaves. We also describe the different shockwave machines currently available and assess their potential clinical utility.

\section{Physics of Waves and Sound Waves:}

A wave is a disturbance in a medium that transports energy without permanently transporting matter. In our context we will be focusing on sound waves, which can be thought of as the propagation of compression followed by relaxation of particles of a medium. An example of a sound wave is the percussion of a drum. When a drum is percussed the vibration of the drum skin induces a disturbance in the molecules of gas (medium) immediately adjacent to the drum skin, which collide with the molecules of gas adjacent to them (compression), but then return to their initial positions (relaxation). Therefore energy, but not mass, is propagated along the medium through a chain reaction of compressions and relaxations as individual particles are displaced from and return to their initial positions

Sound waves are often described by their frequency and amplitude (figure 1). Frequency is the number of times a disturbance is propagated during a specified time interval. Hertz $(\mathrm{Hz})$ is the international system of unit for frequency, which is equal to the number of repeated compressions and relaxations per second. For example, if a drum membrane is percussed once, depending on its tightness, it will create a faster or slower propagation of compression and relaxations. If there are 20 cycles of compression and relaxation in 1 second, its frequency is $20 \mathrm{~Hz}$. If the drum were tighter, it would create a higher number of compressions and relaxations in 1 second and its frequency might be $40 \mathrm{~Hz}$. In human perception of sound, hertz corresponds to pitch.

Sound waves also have an amplitude, which is the maximum displacement from a neutral starting point of a repeating event. In the example of percussion of a drum membrane, this corresponds to the distance displaced by any individual particle from its resting place. This can be measured in any unit of distance. In human perception this corresponds to loudness.

A final important characteristic of a sound wave is the speed of sound in a specific medium. This is independent of frequency or amplitude and is defined by the velocity (or distance traveled per unit of time) of a soundwave in a specific medium. The speed of sound in a medium is affected by the intrinsic stiffness (at a specific temperature) and the density of the medium through which the sound is being propagated. For example, at room temperature (20 C), sound moves through air at 343 Meters/second. Similarly, the speed of sound is different between gases, liquids and solids. For example, when a train is coming, by putting one's ear to the track you can hear the train approaching sooner than by having one's ear to the air, because solids transmit sound faster than gases. A summary of physics terms used so far can be found in Table 1 . 


\section{Different Types of Sound Waves (Ultrasound and Shockwave):}

Sound waves have many important applications in medicine (Table 2). First it is important to note that audible sound waves must have a sufficient amplitude (loudness), and appropriate frequency (between approximately $20-20,000 \mathrm{~Hz}$ ) to be detected by the human ear. An ultrasound wave is a wave that has a frequency above $20,000 \mathrm{~Hz}$, and is therefore not detected by the human ear. However, these high frequency waves are useful because different tissues reflect ultrasound waves differently. In ultrasonography, the reflected ultrasound waves are recorded by transducers and translated into a recorded image.

A shockwave is generated when a wave propagates through a medium at a speed faster than the speed of sound travels through that medium. Shockwaves produce an abrupt spike in pressure over a very short time period (Figure 2). [8] The total energy of the wave is derived from the area under the curve of pressure over time. In shockwave therapy energy flux density to refers to the amount of energy delivered to a specific area of tissue, and therefore can be modulated by changing the peak pressure or area targeted.

\section{Different Types of Shockwaves:}

Delivering shockwaves requires a shockwave generator, and depending on the properties of the shockwave generator, a focuser. Shockwaves are classically generated by three different types of energy sources: electrohydraulic, electromagnetic, or piezoelectric. In electrohydraulic generators, tips of an electrode are submerged in a fluid. When voltage is applied, the fluid is vaporized, which causes rapid expansion in the surrounding fluid leading to shockwave propagation. In electromagnetic generators a fluid is disturbed by applying a voltage across metallic membranes to produce a magnetic field that causes an abrupt movement in a metallic membrane and corresponding shockwave propagation. In pizoelectric generators, piezoceramic elements are embedded into a spherical device submerged in a medium; when voltage is applied the ceramic elements expand, leading to a mechanical disturbance in the surrounding medium. $[9,10]$ In electrohydraulic and most electromagnetic shockwave generators, a lens or reflector is applied to focus the waves at a specific target. However, certain electromagnetic generators and all piezoelectric generators are self-focusing based on the arrangement of the piezoelectric crystals.

Recently, several companies have begun marketing certain devices designated as linear shockwave generators. In this technology shockwaves are focused using an aperture to geometrically-match the shape of corpora and crura. Instead of a point-focuser, these devices deliver energy simultaneously to a larger segment of targeted tissue. [11, 12]

Ballistic radial pressure waves are a fourth type of acoustic wave. They are generated by a controlled explosion leading to an acceleration of a projectile into a probe with the subsequent impact creating a wave. [9] Currently, there are radial pressure wave generating devices that have been marketed for the treatment of ED. Although some describe radial pressure waves as true shockwaves, objectively they are more like "ordinary" sound waves in that they achieve a significantly lower peak pressure, a slower rise time and propagate outwards without a focal point. Additionally, radial pressure wave devices are designated as 
class 1 medical devices, a status given to personal massagers for example, which do not require regulatory approvals and can be used by anyone as no professional training is necessary to administer. In contrast, focused shockwave energy devices described previously are regarded as class 2 medical devices and require pre-market approvals by the FDA by means of clinical safety and effectiveness data.

\section{The Physical Forces Underlying the Biologic Effects of Shockwaves}

Shockwaves are believed to exert physical forces on tissue in two ways. First there is mechanical stress from exposure to the high peak pressure shockwave. Second, in liquids, shockwaves form cavitation bubbles, which are focal areas of vaporization of the liquid. These cavities then collapse when subjected to high pressures, resulting in local trauma and neovascularization. [11] Neovascularization is one of the main hypothesis by which LiESWT exerts its therapeutic effects in ED and has been documented in vivo and in vitro. [13] Other hypothesized mechanisms include the induction of NO [14], nerve regeneration, and stem cell proliferation. $[15,16]$ The exact mechanism by which shockwaves induce these phenomena remains an active area of research. For more information on the biological effects of Li-ESWT we recommend a recently published review by Sokalakis et al. [15]

\section{Available Devices and Clinically Investigated Parameters}

Currently there are several Li-ESWT devices on the market. We include a table summarizing device, wave generator type, treatment protocols based on published randomized controlled trials (RCTs), and their corresponding citations. We have included radial pressure wave therapy devices because they are marketed similarly but have very different characteristics, which we feel is important to differentiate (Table 4).

It is important to point out that follow up data in each of these studies in table 4 is limited to a maximum of 12 months. The Medispec ED1000 device generates electrohydraulic shockwaves and has been investigated with a treatment protocol of 1500 pulses/week for 9 weeks with energy density of $0.09 \mathrm{~mJ} / \mathrm{mm}^{2}$, which demonstrated a statistically significant improvement in International Index of Erectile Function erectile function domain (IIEF-EF) score. [1-4] The Stortz Duolith device generates electromagnetic shockwaves and has been investigated with a treatment protocol of 3000 pulses/week for 5 weeks with energy density of $0.15 \mathrm{~mJ} / \mathrm{mm} 2$, which demonstrated a statistically significant improvement in Erection Hardness Score (EHS) but not IIEF-EF score as compared to placebo. [17] Finally, the Richard-Wolf PiezoWave device generates piezoelectric shockwave and has been investigated with a treatment protocol of 600 pulses/week for 10 weeks with energy density of $0.09 \mathrm{~mJ} / \mathrm{mm}^{2}$, which demonstrated no statistically significant improvement on either EHS or IIEF-EF score. [18] Several other devices are listed in Table 4, which have not been tested in any RCTs. For more comprehensive information regarding randomized controlled trials investigating the efficacy of Li-ESWT for the treatment of ED, please see the meta-analyses by Clavijo et. al [5], Lu et. al [6], the latter of which also contains a systematic review. 


\section{Conclusion}

Despite an extensive literature review, there is still room to understand how Li-ESWT exerts its biological effects. Nonetheless, we feel it important that physicians understand several key variables regarding their shockwave generators before treating patients. Specifically, the type of shockwave generator they are using and the treatment protocols, which have successfully demonstrated efficacy of the device for ED. Finally, given the crucial differences between radial shockwaves and other devices, we feel that it is important to point out that radial shockwaves should not be grouped together with the other shockwave devices and will require their own clinical trials to demonstrate efficacy. This is particularly important because by branding themselves as shockwave therapy, this can confuse both patients and physicians. [19]

\section{References:}

1. Vardi Y, et al., Does low intensity extracorporeal shock wave therapy have a physiological effect on erectile function? Short-term results of a randomized, double-blind, sham controlled study. The Journal of urology, 2012 187(5): p. 1769-1775. [PubMed: 22425129]

2. Yee $\mathrm{CH}$, et al., Extracorporeal shockwave therapy in the treatment of erectile dysfunction: A prospective, randomized, double-blinded, placebo controlled study. International Journal of Urology, 2014 21(10): p. 1041-1045. [PubMed: 24942563]

3. Kitrey ND, et al., Penile low intensity shock wave treatment is able to shift PDE5i nonresponders to responders: a double-blind, sham controlled study. The Journal of urology, 2016 195(5): p. 15501555. [PubMed: 26694904]

4. Srini VS, et al., Low intensity extracorporeal shockwave therapy for erectile dysfunction: a study in an Indian population. Can J Urol, 2015 22(1): p. 7614-7622. [PubMed: 25694008]

5. Clavijo RI, et al., Effects of low-intensity extracorporeal shockwave therapy on erectile dysfunction: a systematic review and meta-analysis. The journal of sexual medicine, 2017 14(1): p. 27-35. [PubMed: 27986492]

6. Lu Z, et al., Low-intensity extracorporeal shock wave treatment improves erectile function: a systematic review and meta-analysis. European urology, 2017 71(2): p. 223-233. [PubMed: 27321373]

7. Rizk PJ, et al., Low-intensity shockwave therapy for erectile dysfunction. Sexual medicine reviews, 2018 6(4): p. 624-630. [PubMed: 29576441]

8. Ramasamy R 2019; Available from: https://clinicaltrials.gov/ct2/show/NCT03670628.

9. McClure S and Dorfmüller C, Extracorporeal shock wave therapy: theory and equipment. Clinical Techniques in Equine Practice, 2003 2(4): p. 348-357.

10. Smith ADP, Glenn M; Kavoussi Louis R; Badlani Gopal H, Smith's Textbook of Endourology. Vol. 12019 691-709.

11. Fode M, et al., Low-intensity shockwave therapy for erectile dysfunction: is the evidence strong enough? Nature Reviews Urology, 2017 14(10): p. 593. [PubMed: 28741629]

12. Bechara A, et al., Twelve-month efficacy and safety of low-intensity shockwave therapy for erectile dysfunction in patients who do not respond to phosphodiesterase type 5 inhibitors. Sexual medicine, 2016 4(4): p. e225-e232. [PubMed: 27444215]

13. Bongrazio M, et al., Shear stress modulates the expression of thrombospondin-1 and CD36 in endothelial cells in vitro and during shear stress-induced angiogenesis in vivo. International journal of immunopathology and pharmacology, 2006 19(1): p. 205873920601900104.

14. Gotte G, et al., Short-time non-enzymatic nitric oxide synthesis from L-arginine and hydrogen peroxide induced by shock waves treatment. FEBS letters, 2002 520(1-3): p. 153-155. [PubMed: 12044888] 
15. Sokolakis I, et al., The Basic Science Behind Low-Intensity Extracorporeal Shockwave Therapy for Erectile Dysfunction: A Systematic Scoping Review of Pre-Clinical Studies. The journal of sexual medicine, 2019 16(2): p. 168-194. [PubMed: 30770067]

16. Behr-Roussel D and Giuliano F, Low-energy shock wave therapy ameliorates erectile dysfunction in a pelvic neurovascular injuries rat model. Translational andrology and urology, 2016 5(6): p. 977. [PubMed: 28078237]

17. Olsen AB, et al., Can low-intensity extracorporeal shockwave therapy improve erectile dysfunction? A prospective, randomized, double-blind, placebo-controlled study. Scandinavian journal of urology, 2015 49(4): p. 329-333. [PubMed: 25470423]

18. Fojecki GL, Tiessen S, and Osther PJ, Effect of low-energy linear shockwave therapy on erectile dysfunction-a double-blinded, sham-controlled, randomized clinical trial. The journal of sexual medicine, 2017 14(1): p. 106-112. [PubMed: 27938990]

19. thriveMD. How Shockwave Therapy for ED Works. 6/2/2019]; Available from: https:// thrivemdvail.com/shockwave-ed-treatment-gainswave-denver-co/.

20. Medispec. 6/2/2019]; Available from: http://www.medispec.com/solutions/solutions_erectiledysfunction/.

21. Tissue Regeneration Technologies.

22. Storz Medical. 6/2/2019]; Available from: https://www.storzmedical.com/en/disciplines/urology/ product-overview/duolith-sd1-ultra-uro.html.

23. Direx Group. 6/3/2019]; Available from: https://www.direxgroup.com/products/erectiledysfunction/morenova.

24. Dornier. 6/3/2019]; Available from: https://www.dornier.com/products/. 


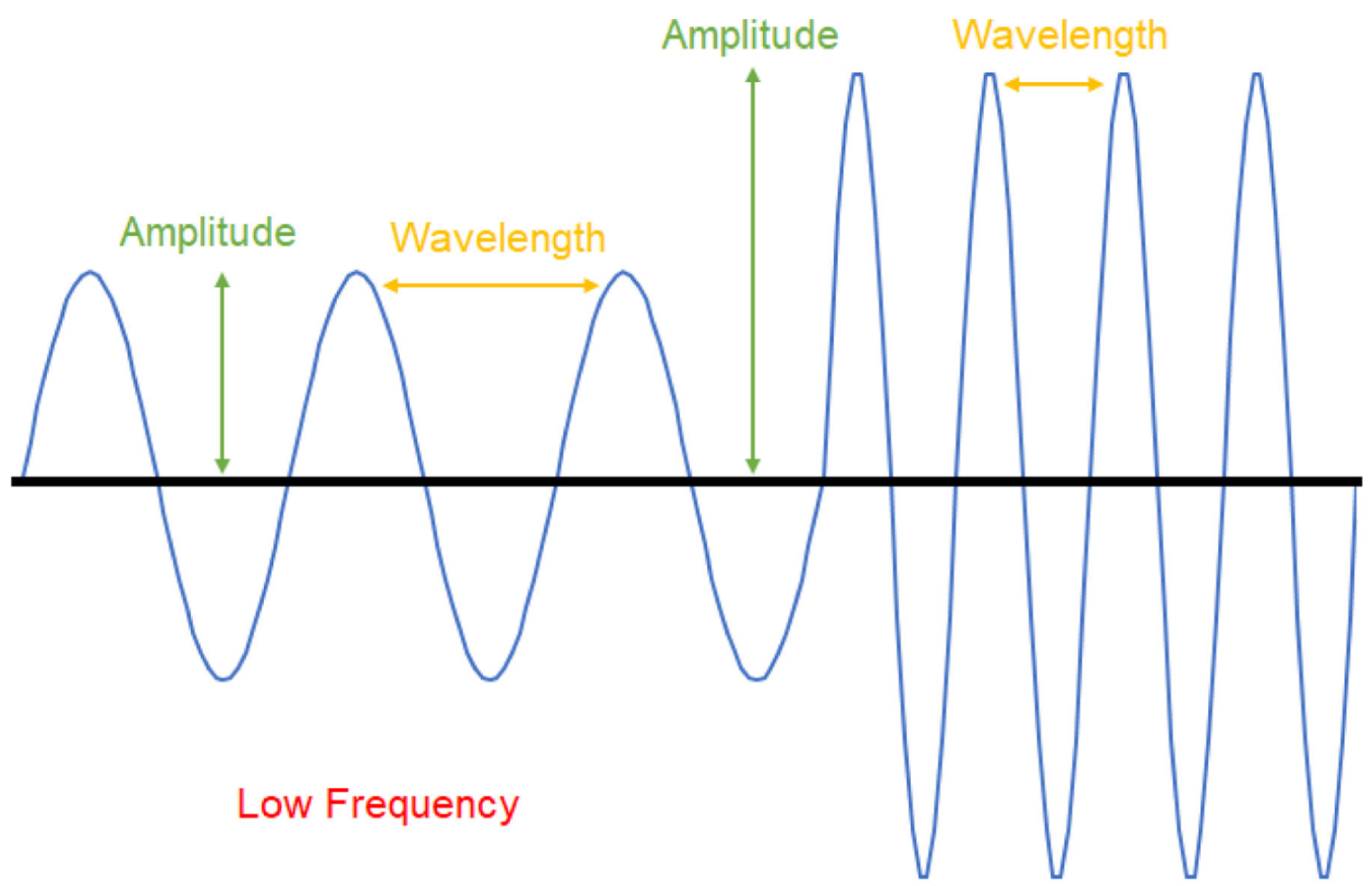

High Frequency

Figure 1.

Figure of Wave with Different Amplitude and Frequency

Example of a wave initially with a low amplitude and low frequency, followed by high amplitude, high frequency. 


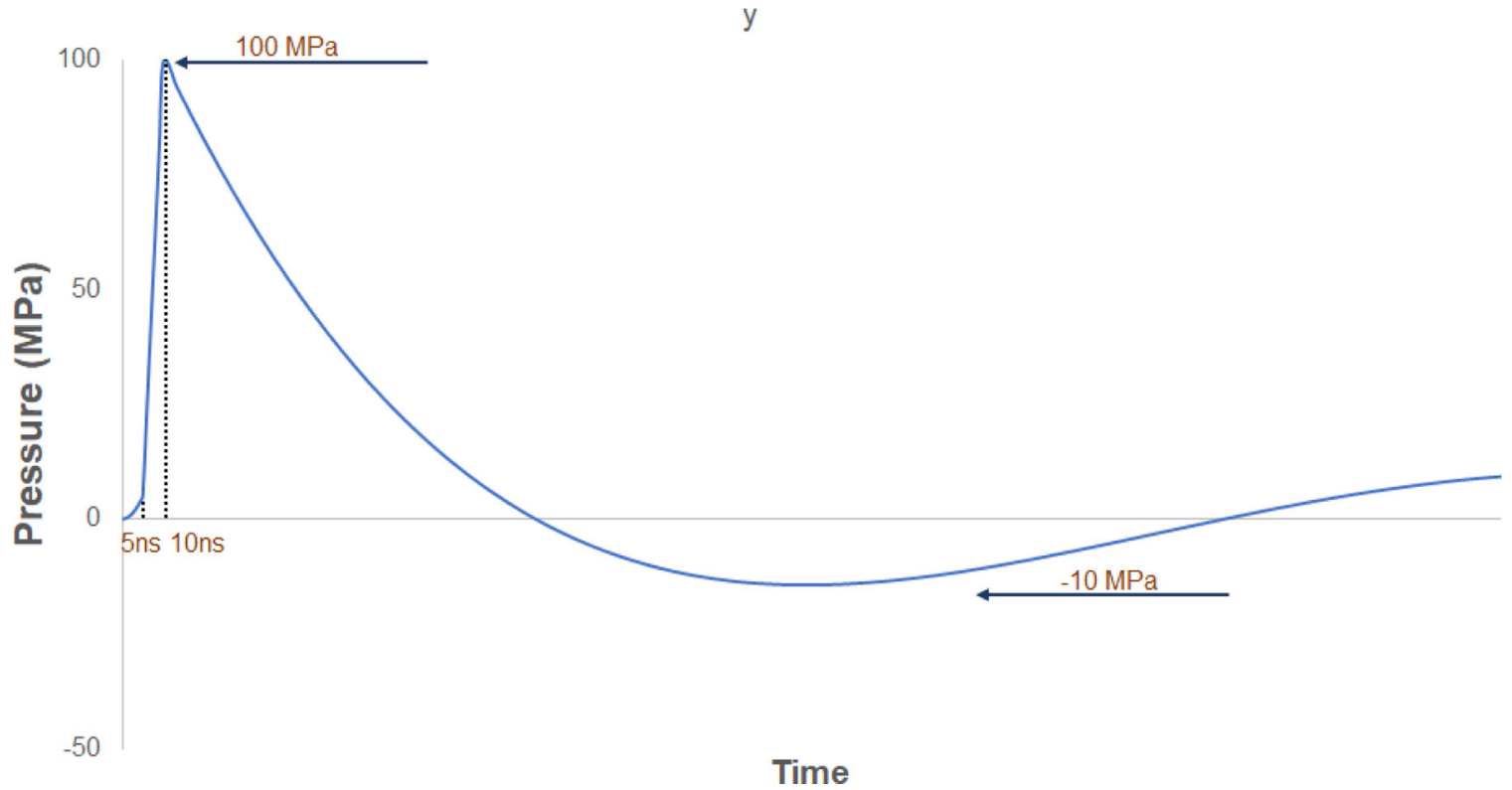

Figure 2.

Pressure vs Time Plot of an Idealized Shockwave

An idealized pressure versus time plot of a shockwave at one specific point along its trajectory. It is noteworthy for its rapid rise time, high positive pressure, and small negative pressure. 


\section{Table 1}

Table of Terms Related to Waves and Wave Characteristics

\begin{tabular}{|l|l|}
\hline Physics Term & Definition \\
\hline Wave & A disturbance in a medium that transports energy without permanently transporting matter. \\
\hline Sound Wave & A propagation of a series of compressions followed by relaxations of particles of a medium such as gas, liquid, solid. \\
\hline Frequency & The number of times a repeating event occurs during a specified time interval. \\
\hline Hertz & The international system unit for frequency, which is equal to the number of repeated events per second. \\
\hline Amplitude & The maximum displacement from a neutral starting point or a repeating event. \\
\hline Wavelength & The distance between repetitions of a wave \\
\hline Speed of Sound & The velocity (or distance traveled per unit of time) of a soundwave in a specific medium. \\
\hline
\end{tabular}


Table 2

Table of Terms Related to Types of Waves

\begin{tabular}{|c|c|c|}
\hline Physics Term & Definition & Example \\
\hline Ultrasound & $\begin{array}{l}\text { Sound waves that have higher frequencies, than } \\
20,000 \mathrm{~Hz} \text {, which is the upper limit perceived by the } \\
\text { human ear }\end{array}$ & $\begin{array}{l}\text { Ultrasound probes generate sound waves at frequencies } \\
\text { higher than } 20,000 \mathrm{~Hz} \text {, and detects the different types of } \\
\text { reflection of the soundwave by the different tissues to } \\
\text { generate an image }\end{array}$ \\
\hline Shockwave & $\begin{array}{l}\text { When a wave propagates through a medium at a speed } \\
\text { faster than the speed of sound travels through that } \\
\text { medium, a shockwave is generated with a characteristic } \\
\text { rapid rise time and high positive peak pressure }\end{array}$ & $\begin{array}{l}\text { When a plane travels faster than the speed of sound through } \\
\text { the air (i.e. faster than } 343 \text { Meters/Second at } 20 \mathrm{C} \text { ), the } \\
\text { acoustic wave generated is a shockwave, experienced as a } \\
\text { sonic boom }\end{array}$ \\
\hline $\begin{array}{l}\text { Energy of a shock } \\
\text { wave }\end{array}$ & $\begin{array}{l}\text { It depends on the peak pressure as well as intrinsic } \\
\text { properties of the medium }\end{array}$ & \\
\hline $\begin{array}{l}\text { Energy Flux } \\
\text { Density (EFD) }\end{array}$ & The rate of energy transfer per unit area i.e. $\mathrm{J} / \mathrm{S} / \mathrm{M}^{2}$ & $\begin{array}{l}\text { A high pressure or energy shockwave delivered to a small } \\
\text { area will have a higher EFD than the same shockwave } \\
\text { delivered to a larger area. }\end{array}$ \\
\hline
\end{tabular}


Table 3

\section{Different Types of Shockwaves}

\begin{tabular}{|l|l|}
\hline Physics Term & Mechanism \\
\hline $\begin{array}{l}\text { Electrohydraulic } \\
\text { shockwave }\end{array}$ & $\begin{array}{l}\text { Tips of an electrode are submerged in a fluid, when voltage is applied the fluid is vaporized which causes rapid } \\
\text { expansion in the surrounding fluid leading to shockwave propagation. }\end{array}$ \\
\hline $\begin{array}{l}\text { Electromagnetic } \\
\text { shockwave }\end{array}$ & $\begin{array}{l}\text { Fluid is disturbed by applying a voltage across metallic membranes to produce a magnetic field that causes an } \\
\text { abrupt movement in a metallic membrane and corresponding shockwave propagation }\end{array}$ \\
\hline Pizoelectric shockwave & $\begin{array}{l}\text { Piezoceramic elements are embedded into a spherical device submerged in a medium; when voltage is applied to } \\
\text { the ceramic elements expand, inducing a mechanical disturbance in the medium resulting in shockwave } \\
\text { propagation }\end{array}$ \\
\hline Linear Li-ESWT & $\begin{array}{l}\text { Shockwave focused over a larger surface area, designed to conform to more linear tissue such as the corpora/ } \\
\text { crura. }\end{array}$ \\
\hline Radial waves & A controlled explosion of compressed air bursts a projectile into a probe, which deforms creating a wave \\
\hline
\end{tabular}




\section{Table 4}

Summary of Devices Currently Available

\begin{tabular}{|c|c|c|}
\hline Applicator & Wave Type & Treatment Protocols for ED Based on RCTs \\
\hline Medispec ED1000 [17] & $\begin{array}{l}\text { Electrohydraulic } \\
\text { Shockwave }\end{array}$ & $\begin{array}{l}\text { Effective Treatment Protocol: } \\
\bullet 1500 \text { pulses/week for } 9 \text { weeks with energy density of } 0.09 \mathrm{~mJ} / \mathrm{mm}^{2}[1- \\
4] \\
\text { Ineffective Treatment Protocol: } \\
\cdot \text { N/A }\end{array}$ \\
\hline Stortz Duolith [18] & $\begin{array}{l}\text { Electromagnetic } \\
\text { Shockwave }\end{array}$ & $\begin{array}{l}\text { Effective Treatment Protocol: } \\
\text { • } 3000 \text { pulses/week for } 5 \text { weeks with energy density of } 0.15 \mathrm{~mJ} / \mathrm{mm}^{2} \text { [19] } \\
\text { Ineffective Treatment Protocol: } \\
\text { • N/A }\end{array}$ \\
\hline Direx Renova and MoreNova [20] & Electromagnetic & None \\
\hline Dornier Dornier Aries 2 [21] & Electromagnetic & None \\
\hline Richard-Wolf PiezoWave [22] & Piezoelectric-Shockwave & $\begin{array}{l}\text { Ineffective Treatment Protocol: } \\
\cdot 600 \text { pulses/week for } 10 \text { weeks with energy density of } 0.09 \mathrm{~mJ} / \mathrm{mm}^{2}[23]\end{array}$ \\
\hline Gainswave & Radial Waves & None \\
\hline
\end{tabular}

